\title{
Acute effects of Radiotherapy in patients with Head and Neck Cancer: IMRT vS RapidArc $^{\mathrm{TM}}$
}

\author{
Efeitos Agudos do Tratamento de Radioterapia em Doentes com Carcinoma de Cabeça e \\ Pescoço: IMRT vs RapidArc ${ }^{T M}$
}

\author{
Liliana Silva ${ }^{1}$, Bárbara Barbosa ${ }^{2}$, Marisa Matos ${ }^{3}$, \\ ${ }^{1}$ School of Health and Technology of Porto, Instituto Politécnico do Porto; \\ ${ }^{2}$ Radiotherapy Service of the Portuguese Institute of Oncology Francisco Gentil, E.P.E; \\ ${ }^{3}$ Nursing Service of the Portuguese Institute of Oncology Francisco Gentil, E.P.E \\ e-mail: li.silva.gmr@gmail.com
}

\begin{abstract}
Radiation therapy is a standard treatment in the multidisciplinary approach to the treatment of head and neck carcinomas (HNC). The description and perception of the effects caused by the treatment in the normal tissues is important for the clinical evaluation of the patient because treatment is interrupted if the patient develops several acute side effects which directly affect their survival. This study involved 46 patients over 6 weeks of treatment and evaluated the observed acute effects of treatment in five different anatomical areas - skin, mucosa, salivary glands, pharynx /esophagus and larynx. This evaluation adopted the classification of the Acute Radiation Morbidity Scoring Criteria of the Radiation Therapy Oncology Group (RTOG).

Data analysis revealed that the acute effects related to the RapidArc ${ }^{\mathrm{TM}}$ technique appeared earlier when compared to IMRT techniques. On the other hand, the IMRT technique showed more exacerbated high-grade effects.
\end{abstract}

Keywords: RT, IMRT, RapidArc ${ }^{\mathrm{TM}}$, Effects, RTOG

\section{Resumo}

A Radioterapia é parte integrante, standart, da abordagem multidisciplinar do tratamento de Carcinomas de Cabeça e Pescoço (CPP). A perceção e descrição dos efeitos provocados pelo tratamento ao nível dos tecidos normais é importante para avaliação clínica do doente, já que com o aparecimento de efeitos agudos severos/graves ao tratamento, este é interrompido, o que afetando diretamente a sobrevida do doente. Este estudo incidiu na avaliação de 6 semanas de tratamento de 46 doentes, contemplando a avaliação dos efeitos agudos do tratamento em cinco áreas anatómicas distintas: Pele, Mucosa, Glândulas Salivares, Faringe/Esófago e Laringe. Fundamentada nos critérios de classificação do Acute Radiation Morbidity Scoring Criteria da Radiation Therapy Oncology Group (RTOG).

Após a avaliação e análise dos dados, verificou-se que os efeitos agudos obtidos com a técnica de RapidArc ${ }^{\mathrm{TM}}$ surgiram mais precocemente, quando comparados com técnica de IMRT. Por outro lado, com a técnica de IMRT registaram-se efeitos mais exacerbados, de alto Grau.

Palavras-Chave: RT, IMRT, RapidArc ${ }^{\mathrm{TM}}$, Efeitos, RTOG 


\section{Introduction}

Radiotherapy (RT) is a therapeutic modality that uses radiation for the treatment of cancer. It is applied with an appropriate predetermined dose to the target in order to spare the healthiest tissues adjacent to the lesion ${ }^{11,2}$. Head and neck cancer (HNC) includes any tumor in the upper aerodigestive epithelium, as tumors in Peri-Nasal Sinuses, Nasal Cavity, Oral Cavity, Salivary Glands, Pharynx and Larynx. These tumors might show an aggressive biological behavior and sometimes may easily be visible and palpable ${ }^{7}$

The HNC appears as the $6^{\text {th }}$ most common carcinoma worldwide, accounting for $6 \%$ of all diagnosed cancers 1. In Portugal, the Larynx Carcinoma is located in the 10 most frequent carcinomas in men, and consequently in the top 10 causes of cancer death in men ${ }^{14}$. Men are most commonly affected, although the incidence in women is increasing because of changes in lifestyle. In the records of the 2007 North Regional Cancer Registry (RORENO), the incidence of carcinomas of the oral cavity and pharynx in men was $25,7 \%$ (409 cases reported) compared with the $5,2 \%$ incidence rate in women (89 cases reported) (RORENO, 2007). It is estimated that $75 \%$ of patients are regular consumers of alcohol and tobacco. People who have never smoked but who are substantial consumers of alcohol have an increased risk of developing this type of lesions ${ }^{10}$.

The therapeutic approaches of this type of lesions include standard practices such as surgery and radiotherapy (RT) and may be combined with chemotherapy (CT) 3. The prescription dose required for treatment is based on several factors, including the location, the type of lesion, the aim of treatment, and can be combined with other types of treatment modalities. When the treatment has a curative approach the dose is between 50 Gy and $70 \mathrm{~Gy}$, for a total treatment time of between 5-7 weeks in conventional fractionation $(1,8 \mathrm{~Gy} / \mathrm{d}$ to $2 \mathrm{~Gy} / \mathrm{d})$ or in altered fractionation schedules ${ }^{18}$.

Fast advances in radiation therapy techniques allow a substantial amount of the normal tissues to be spared; however, most patients treated with curative doses will develop acute side effects to the treatment, which require an intervention of multidisciplinary specialized support. Nevertheless, most of these effects are temporary ${ }^{5,6}$.

In the irradiation of the complex area of the head and neck region, the damage to normal tissues becomes evident in cases of serious complications. The patient is unable to continue therapy due to the appearance of several acute effects as a result of the treatment. This interruption directly affects the survival and effective-

\section{Introdução}

A Radioterapia (RT) é uma modalidade terapêutica que utiliza feixes de radiação ionizante para o tratamento de lesões malignas e benignas, com o objetivo de irradiar uma lesão alvo com uma dose pré-determinada a um volume conformado, poupando ao máximo os tecidos saudáveis adjacentes à lesão ${ }^{11,2}$.

Designa-se por "Carcinoma de Cabeça e Pescoço" (CCP) qualquer tumor que surja localizado na parte superior do epitélio aerodigestivo. Estes tumores podem apresentar um comportamento biológico agressivo, sendo, por vezes, facilmente observados e palpados. Temos como exemplo destes tumores os Seios Peri-nasais, Cavidade Nasal, Cavidade Oral, Glândulas Salivares, Faringe e Laringe ${ }^{7}$.

O CCP apresenta-se como a $6^{\mathrm{a}}$ neoplasia mais frequente em todo o mundo, representando $6 \%$ de todas as neoplasias diagnosticadas ${ }^{1}$. Em Portugal, o Carcinoma da Laringe encontra-se entre os 10 carcinomas mais frequentes no homem e consequentemente entre as 10 principais causas de morte por cancro no homem ${ }^{14}$. Os homens são mais frequentemente afetados, embora a incidência nas mulheres esteja a aumentar devido às mudanças do estilo de vida. Nos registos de 2007 do Registo Oncológico Regional do Norte (RORENO), a taxa de incidência de Carcinomas da Cavidade Oral e Faringe no homem foi de 25,7\% (409 casos registados) comparativamente com a taxa de incidência nas mulheres que foi de 5,2 \% (89 casos registados) (RORENO, 2007). Estima-se que 75 $\%$ dos doentes sejam consumidores de tabaco e álcool regularmente. Pessoas que nunca fumaram, mas que sejam consumidores substanciais de álcool têm um risco aumentado de desenvolver este tipo de lesão ${ }^{10}$.

As abordagens terapêuticas deste tipo de lesões incluem como práticas standard a Cirurgia e a Radioterapia (RT) e podem ser conjugadas com esquemas de Quimioterapia $(\mathrm{QT})^{3}$. A prescrição da dose necessária para o tratamento é baseada em diversos fatores, nomeadamente na localização, no tipo de lesão, no intuito do tratamento, no facto de ser combinada com outras modalidades de tratamento, entre outros. Quando o intuito é curativo, as doses podem variar entre os 50 Gy e os $70 \mathrm{~Gy}$, perfazendo um tempo total de tratamento de 5 a 7 semanas, em esquemas de fracionamento convencional (1,8 Gy/d a $2 \mathrm{~Gy} / \mathrm{d})$ ou em esquemas alterados de fracionamento ${ }^{18}$.

Os rápidos avanços nas técnicas de Radioterapia permitem uma poupança considerável dos tecidos normais, no entanto, a maioria dos doentes tratados com doses curativas desenvolverão efeitos agudos ao tratamento, o que requererá a intervenção de um apoio multidisciplinar especializado. No entanto, a maioria destes efeitos assume 
ness of the therapy. Several prospective studies have demonstrated an increase in acute side effects of RT treatment in patients with concomitant chemotherapy (CT) ${ }^{17}$.

Thus, certain studies have been performed and technical advances in RT have been achieved, especially in the intensity-modulated techniques such as IMRT and RapidArc $^{\mathrm{TM}}$. These have led to sophisticated tools and treatment planning systems which in the end contribute to the reduction of some side effects of treatment, essentially in patients treated with RT or concomitant $\mathrm{CT}^{4}$. Therefore, it is necessity to expand the identification and differentiation of treatment of acute effects with these two techniques in order to make a direct comparison between techniques in terms of biological manifestations. Effects evaluation was based on the criteria of the Radiation Therapy Oncology Group (RTOG).

\section{Materials and Methods}

The data collection was performed at the Department of Radiotherapy, in the Portuguese Oncology Institute of Porto, Francisco Gil E.P.E., between $21^{\text {st }}$ November 2011 and $19^{\text {th }}$ May 2012. A total of 46 patients were included in the study. The selection criteria were based on the pathology of the patient (patients with only HNC); localization of lesion; the RT technique used in treatment (IMRT or RapidArc ${ }^{\mathrm{TM}}$ ); the aim of treatment (we excluded patients with $\mathrm{HNC}$ in palliative treatment) and signed informed consent.

The choice of RT treatment techniques took into account the treatment protocols used in the institution. Of the 46 patients who started the study, 42 were male and 4 female. The selected patients were divided into 2 groups: Group 1 included 13 patients who were submitted to the IMRT technique, and in Group 2, 33 patients were submitted to treatment with the RapidArc ${ }^{\mathrm{TM}}$ technique. Of the total sample, 13 patients (4 in Group 1 and 9 in Group 2) were submitted to concomitant treatment with CT. It is also important to mention that 14 of the 46 patients that started the study underwent RT treatment with a non-conventional fractionation. This was Simultaneous Integrated Boost (SIB) for clinical orientation, which determines the total time of treatment (there is a um comportamento temporário ${ }^{5,6}$. Na irradiação da região de Cabeça e Pescoço os danos nos tecidos normais tornam-se evidentes, principalmente por se tratar de uma área complexa (RTOG), em casos de complicações graves, ou seja, de aparecimento de efeitos agudos severos ao tratamento, este é interrompido por incapacidade do doente em continuar a terapia, o que afeta diretamente a sobrevida e a própria eficácia da terapia na procura pela cura do doente. Vários estudos prospetivos têm demostrado o aumento dos efeitos agudos, ao tratamento de RT, em doentes que estejam a realizar QT concomitante ${ }^{17}$. Desta forma, têm sido realizados alguns estudos e avanços nas técnicas de RT, nomeadamente com o aparecimento das técnicas de intensidade modelada como a IMRT e o RapidArc ${ }^{\mathrm{TM}}$, que têm permitido a criação de sofisticadas ferramentas de planeamento do tratamento e de sistemas, o que culmina na redução de alguns efeitos secundários, principalmente por parte de doentes tratados com RT ou com QT concomitante ${ }^{4}$. Portanto, surgiu a necessidade de aprofundar a identificação e diferenciação dos efeitos agudos ao tratamento com estas duas técnicas, por forma a realizar uma comparação direta entre as duas técnicas do ponto de vista das manifestações biológicas. Ambas foram utilizadas com base os critérios da Radiation Therapy Oncology Group (RTOG) para a respectiva avaliação.

\section{Materiais e Métodos}

A recolha de dados foi realizada no Serviço de Radioterapia do Instituto Português de Oncologia do Porto, Francisco Gil, E.P.E, entre 21 de Novembro de 2011 e 19 de Maio de 2012, com um total de 46 doentes em estudo. Os critérios de seleção para amostragem foram: a patologia do doente (doentes unicamente com CCP), localização da lesão, a técnica de RT utilizada (IMRT ou RapidArc ${ }^{\mathrm{TM}}$ ), intuito do tratamento (foram excluídos os doentes com Carcinoma de Cabeça e Pescoço a realizar tratamento Paliativo) e assinatura do Consentimento Informado. A escolha das técnicas de RT tiveram em conta os protocolos de tratamento utilizados no serviço em questão, visto que estas são as duas técnicas utilizadas. Dos 46 doentes que iniciaram o estudo, 42 eram do sexo masculino e 4 do sexo feminino. Os doentes selecionados foram divididos em 2 grupos: o Grupo 1, constituído por 13 doentes que realizaram a técnica IMRT; e o Grupo 2, 33 doentes que realizaram tratamento com a técnica de RapidArc ${ }^{\mathrm{TM}}$. Do universo total da amostra, 13 doentes (4 do Grupo 1 e 9 do Grupo 2) estavam a realizar tratamento com QT concomitante. É, também, importante referir que 14 dos 46 doentes que iniciaram o estudo, realizaram tratamento de RT com um fracionamento não convencional, ou seja, com Simultaneous Integrated Boost (SIB), 
reduction) and an increase in dose per fraction. The variables under observation in this study were the acute effects registered at five anatomical areas based on the RTOG guidelines system for the HNC indicated in Table 1.

Table 1: Acute Radiation Morbidity Scoring Criteria

\begin{tabular}{|c|c|c|c|c|c|}
\hline & Skin & Mucosa & Salivary Glands & Pharynx / Esophagus & Larynx \\
\hline Grade 0 & $\begin{array}{c}\text { No change over } \\
\text { baseline }\end{array}$ & $\begin{array}{c}\text { No change over } \\
\text { baseline }\end{array}$ & $\begin{array}{l}\text { No change over } \\
\text { baseline }\end{array}$ & No change over baseline & $\begin{array}{l}\text { No change over } \\
\text { baseline }\end{array}$ \\
\hline Grade 1 & $\begin{array}{l}\text { Follicular, faint } \\
\text { or dull erythema/ } \\
\text { epilation/dry } \\
\text { desquamation/ } \\
\text { decreased } \\
\text { sweating }\end{array}$ & $\begin{array}{c}\text { Injection/ may } \\
\text { experience mild } \\
\text { pain not requiring } \\
\text { analgesic }\end{array}$ & $\begin{array}{l}\text { Mild mouth dryness/ } \\
\text { slightly thickened } \\
\text { saliva/ may have } \\
\text { slightly altered taste } \\
\text { such as metallic taste/ } \\
\text { these changes not } \\
\text { reflected in alteration } \\
\text { in baseline feeding } \\
\text { behavior, such as } \\
\text { increased use of } \\
\text { liquids with meals }\end{array}$ & $\begin{array}{c}\text { Mild dysphagia or } \\
\text { odynophagia/ may } \\
\text { require topical anesthetic } \\
\text { or non-narcotic } \\
\text { analgesics/ may require } \\
\text { soft diet }\end{array}$ & $\begin{array}{l}\text { Mild or intermittent } \\
\text { hoarseness/cough not } \\
\text { requiring antitussive/ } \\
\text { erythema of mucosa }\end{array}$ \\
\hline Grade 2 & $\begin{array}{l}\text { Tender or bright } \\
\text { erythema, } \\
\text { patchy moist } \\
\text { desquamation/ } \\
\text { moderate edema }\end{array}$ & $\begin{array}{l}\text { Patchy mucositis } \\
\text { which may } \\
\text { produce an } \\
\text { inflammatory } \\
\text { serosanguinitis } \\
\text { discharge/ may } \\
\text { experience } \\
\text { moderate } \\
\text { pain requiring } \\
\text { analgesia }\end{array}$ & $\begin{array}{l}\text { Moderate to complete } \\
\text { dryness/ thick, sticky } \\
\text { saliva/ markedly } \\
\text { altered taste }\end{array}$ & $\begin{array}{l}\text { Moderate dysphagia } \\
\text { or odynophagia/ } \\
\text { may require narcotic } \\
\text { analgesics/ may require } \\
\text { puree or liquid diet }\end{array}$ & $\begin{array}{l}\text { Persistent hoarseness } \\
\text { but able to vocalize/ } \\
\text { referred ear pain, } \\
\text { sore throat, patchy } \\
\text { fibrinous exudate or } \\
\text { mild arytenoid edema } \\
\text { not requiring narcotic/ } \\
\text { cough requiring } \\
\text { antitussive }\end{array}$ \\
\hline Grade 3 & $\begin{array}{l}\text { Confluent, moist } \\
\text { desquamation } \\
\text { other than skin } \\
\text { folds, pitting } \\
\text { edema }\end{array}$ & $\begin{array}{c}\text { Confluent } \\
\text { fibrinous } \\
\text { mucositis/ may } \\
\text { include severe } \\
\text { pain requiring } \\
\text { narcotic }\end{array}$ & ------- & $\begin{array}{l}\text { Whispered speech, throat } \\
\text { pain or referred ear } \\
\text { pain requiring narcotic/ } \\
\text { confluent fibrinous } \\
\text { exudate, marked } \\
\text { arytenoid edema }\end{array}$ & $\begin{array}{l}\text { Whispered speech, } \\
\text { throat pain or referred } \\
\text { ear pain requiring } \\
\text { narcotic/ confluent } \\
\text { fibrinous exudate, } \\
\text { marked arytenoid } \\
\text { edema }\end{array}$ \\
\hline Grade 4 & $\begin{array}{l}\text { Ulceration, } \\
\text { hemorrhage, } \\
\text { necrosis }\end{array}$ & $\begin{array}{l}\text { Ulceration, } \\
\text { hemorrhage or } \\
\text { necrosis }\end{array}$ & $\begin{array}{c}\text { Acute salivary gland } \\
\text { necrosis }\end{array}$ & $\begin{array}{l}\text { Complete obstruction, } \\
\text { ulceration, perforation, } \\
\text { fistula }\end{array}$ & $\begin{array}{l}\text { Marked dyspnea, } \\
\text { stridor or hemoptysis } \\
\text { with tracheostomy or } \\
\text { intubation necessary }\end{array}$ \\
\hline
\end{tabular}

Observation and registration of the effects were carried out for both study groups, from the first week of treatment until the end. The evaluation period changed within the sample group because of the individual nature of the dosimetric plane which was conditioned by treatment dose and the fractionation schedules. The data were statistically treated using the Statistical Package for Social Science (SPSS), version 18.0, using descriptive techniques such as absolute frequencies (number) and relative frequencies (\%), which are represented in comparative frequency tables and normalized to total number of patients per treatment technique. 
por orientação clínica, o que condiciona o tempo total de tratamento (há uma redução do mesmo) e um aumento da dose por fração.

As variáveis em observação neste estudo foram os efeitos secundários verificados ao nível de 5 áreas anatómicas com base no sistema de classificação da RTOG, para o CCP, indicados na tabela 1 .

Tabela 1: Critérios de valoração da borbilidade de exposição aguda à radiação

\begin{tabular}{|c|c|c|c|c|c|}
\hline & Pele & Mucosa & Glândulas Salivares & Faringe/ Esófago & Laringe \\
\hline Grau 0 & $\begin{array}{l}\text { Nenhuma } \\
\text { mudança em } \\
\text { relação ao } \\
\text { início }\end{array}$ & $\begin{array}{l}\text { Nenhuma } \\
\text { mudança em } \\
\text { relação ao } \\
\text { início }\end{array}$ & $\begin{array}{l}\text { Nenhuma mudança em } \\
\text { relação ao início }\end{array}$ & $\begin{array}{l}\text { Nenhuma mudança em } \\
\text { relação ao início }\end{array}$ & $\begin{array}{l}\text { Nenhuma mudança em } \\
\text { relação ao início }\end{array}$ \\
\hline Grau 1 & $\begin{array}{l}\text { Folicular, fraco } \\
\text { ou eritema } \\
\text { maçante / } \\
\text { depilação / } \\
\text { descamação } \\
\text { seca / } \\
\text { diminuição da } \\
\text { transpiração }\end{array}$ & $\begin{array}{l}\text { Injeção / } \\
\text { pode sentir } \\
\text { dor leve sem } \\
\text { necessidade } \\
\text { de analgesia }\end{array}$ & $\begin{array}{l}\text { Boca suavemente seca/ } \\
\text { saliva espessa / pode ter } \\
\text { ligeiramente alterado gosto } \\
\text { como gosto metálico / sem } \\
\text { alterações de alimentação } \\
\text { da linha de base, como o } \\
\text { aumento do uso de líquidos } \\
\text { durante as refeições }\end{array}$ & $\begin{array}{l}\text { Disfagia leve ou odinofagia } \\
\text { / pode exigir anestésicos ou } \\
\text { analgésicos não-narcóticos } \\
\text { / pode exigir dieta leve }\end{array}$ & $\begin{array}{l}\text { Leve ou intermitente } \\
\text { rouquidão / tosse sem } \\
\text { necessidade de medicação/ } \\
\text { eritema da mucosa }\end{array}$ \\
\hline Grau 2 & $\begin{array}{c}\text { Eritema } \\
\text { brilhante, } \\
\text { coceira } \\
\text { descamação } \\
\text { húmida / edema } \\
\text { moderado }\end{array}$ & $\begin{array}{l}\text { Mucosite } \\
\text { irregular que } \\
\text { pode produzir } \\
\text { hemorragias } \\
\text { / pode sentir } \\
\text { dor moderada } \\
\text { necessitando } \\
\text { de analgesia }\end{array}$ & $\begin{array}{l}\text { Moderada à secura total / } \\
\text { espessura, saliva pegajosa } \\
\text { / gosto marcadamente } \\
\text { alterada }\end{array}$ & $\begin{array}{l}\text { Disfagia moderada ou } \\
\text { odinofagia / pode exigir } \\
\text { analgésicos narcóticos } \\
\text { / pode exigir dieta } \\
\text { semilíquida ou liquida }\end{array}$ & $\begin{array}{l}\text { Rouquidão persistente, mas } \\
\text { capaz de vocalizar / dor de } \\
\text { ouvido, dor de garganta, } \\
\text { exsudato fibrinoso irregular } \\
\text { ou edema das aritenóides, } \\
\text { sem necessidade de } \\
\text { medicação/ tosse exigindo } \\
\text { antitussíco }\end{array}$ \\
\hline Grau 3 & $\begin{array}{c}\text { Confluentes, } \\
\text { descamação } \\
\text { húmida } \\
\text { diferente } \\
\text { dobras da } \\
\text { pele, edema da } \\
\text { picada }\end{array}$ & $\begin{array}{l}\text { Mucosite } \\
\text { fibrinosa } \\
\text { confluente } \\
\text { / podem } \\
\text { incluir dor } \\
\text { necessitando } \\
\text { de analgesia }\end{array}$ & -------- & $\begin{array}{c}\text { Disfagia moderada a } \\
\text { severa ou odinofagia com } \\
\text { desidratação ou perda } \\
\text { de peso ( }>15 \% \text { da linha } \\
\text { de base pré-tratamento) } \\
\text { requerendo tubo de } \\
\text { alimentação nasogástica, } \\
\text { / analgesia / pode exigir } \\
\text { semilíquida ou liquida }\end{array}$ & $\begin{array}{l}\text { Fala sussurrada, dor de } \\
\text { garganta ou dor de ouvido } \\
\text { necessitando de analgesia } \\
\text { / confluente exsudato } \\
\text { fibrinoso, marcado edema } \\
\text { aritenóide }\end{array}$ \\
\hline Grau 4 & $\begin{array}{c}\text { Ulceração, } \\
\text { hemorragia ou } \\
\text { necrose }\end{array}$ & $\begin{array}{l}\text { Ulceração, } \\
\text { hemorragia } \\
\text { ou necrose }\end{array}$ & Necrose aguda & $\begin{array}{l}\text { Obstrução completa, } \\
\text { ulceração, perfuração, } \\
\text { fístula }\end{array}$ & $\begin{array}{c}\text { Dispneia marcada, } \\
\text { hemoptise com } \\
\text { traqueostomia e intubação }\end{array}$ \\
\hline
\end{tabular}

A observação e o registo dos efeitos foram realizados para ambos os grupos em estudo, desde a primeira semana de tratamento do doente até ao seu término. O período de avaliação variou na amostragem, devido à individualidade de cada plano dosimétrico condicionado pela dose de tratamento e pelo esquema de fracionamento. Os dados obtidos foram tratados estatisticamente através do programa Statistical Package for the Social Science (SPSS), versão 18.0, recorrendo a técnicas descritivas tais como as frequências absolutas $\left(\mathrm{n}^{\circ}\right)$ e frequências relativas (\%), representados em tabelas de frequências comparativas, normalizados ao número total de doentes por técnica, e representados graficamente. 


\section{Results}

Table 2: Results observed in Group 1 (IMRT) in different structures in evaluation during the treatment weeks

\begin{tabular}{|c|c|c|c|c|c|c|c|}
\hline \multirow{2}{*}{$\begin{array}{c}\text { Structure in } \\
\text { Evaluation }\end{array}$} & \multirow{2}{*}{ Grade } & \multicolumn{6}{|c|}{ Weeks of treatment } \\
\hline & & $\mathbf{1}^{\mathrm{a}}$ & $2^{a}$ & $3^{a}$ & $4^{a}$ & $5^{a}$ & $6^{\mathrm{a}}$ \\
\hline \multirow{6}{*}{ Skin } & Grade 0 & 11 & 4 & 1 & - & - & - \\
\hline & Grade 1 & 2 & 8 & 6 & 2 & 2 & 1 \\
\hline & Grade 2 & - & 1 & 5 & 6 & 7 & 9 \\
\hline & Grade 3 & - & - & 1 & 5 & 3 & 1 \\
\hline & Grade 4 & - & - & - & - & 1 & 1 \\
\hline & Total & 13 & 13 & 13 & 13 & 13 & 12 \\
\hline \multirow{6}{*}{ Mucosa } & Grade 0 & 11 & 2 & - & - & - & - \\
\hline & Grade 1 & 1 & 7 & 6 & 1 & 1 & 2 \\
\hline & Grade 2 & 1 & 4 & 3 & 7 & 6 & 4 \\
\hline & Grade 3 & - & - & 4 & 4 & 4 & 4 \\
\hline & Grade 4 & - & - & & 1 & 2 & 2 \\
\hline & Total & 13 & 13 & 13 & 13 & 13 & 12 \\
\hline \multirow{6}{*}{$\begin{array}{c}\text { Salivary } \\
\text { Glands }\end{array}$} & Grade 0 & 11 & 6 & 1 & - & - & - \\
\hline & Grade 1 & 2 & 6 & 8 & 4 & 2 & - \\
\hline & Grade 2 & - & 1 & 3 & 8 & 10 & 2 \\
\hline & Grade 3 & - & - & 1 & 1 & - & 9 \\
\hline & Grade 4 & - & - & - & - & 1 & 1 \\
\hline & Total & 13 & 13 & 13 & 13 & 13 & 12 \\
\hline \multirow{6}{*}{$\begin{array}{l}\text { Pharynx / } \\
\text { Esophagus }\end{array}$} & Grade 0 & 9 & 6 & 3 & 1 & 1 & 1 \\
\hline & Grade 1 & 3 & 5 & 7 & 6 & 4 & 3 \\
\hline & Grade 2 & 1 & 1 & 2 & 4 & 5 & 4 \\
\hline & Grade 3 & - & - & - & 1 & 2 & 3 \\
\hline & Grade 4 & - & - & 1 & 1 & 1 & 1 \\
\hline & Total & 13 & 13 & 13 & 13 & 13 & 12 \\
\hline \multirow{6}{*}{ Larynx } & Grade 0 & 11 & 10 & 9 & 8 & 8 & 7 \\
\hline & Grade 1 & 2 & 3 & 3 & 3 & 1 & 1 \\
\hline & Grade 2 & - & - & 1 & 2 & 4 & 3 \\
\hline & Grade 3 & - & - & - & - & - & 1 \\
\hline & Grade 4 & - & - & - & - & - & - \\
\hline & Total & 13 & 13 & 13 & 13 & 13 & 12 \\
\hline
\end{tabular}

During the evaluation period, 1 patient in Group 2 (RapidArc ${ }^{\mathrm{TM}}$ ) dropped out between the $3^{\text {rd }}$ and $4^{\text {th }}$ week for reasons unrelated to the treatment. Between the $5^{\text {th }}$ and $6^{\text {th }}$ weeks, 1 patient in Group 1 and 14 patients in Group 2 completed their treatment from a total of 30 patients. The remaining patients continued under evaluation in the $6^{\text {th }}$ week. Tables 2 and 3 represent the patients' evolution which is evaluated in terms of the registered acute effects according to the particular technique applied.

Graphs are presented to facilitate data interpretation (Figure 1). All patients in Group 1 developed skin side effects in week 4, while in Group 2, this only occurred from week 5 onwards. In week 5, Grade 4 effects
Resultados

Tabela 2: Resultados observados no Grupo 1 (IMRT), nas diferentes estruturas em avaliação durante as semanas de tratamento

\begin{tabular}{|c|c|c|c|c|c|c|c|}
\hline \multirow{2}{*}{$\begin{array}{c}\text { Estrutura } \\
\text { em Avaliação }\end{array}$} & \multirow{2}{*}{ Graus } & \multicolumn{6}{|c|}{ Semanas de tratamento } \\
\hline & & $\mathbf{1}^{\mathrm{a}}$ & $2^{\mathrm{a}}$ & $3^{\mathbf{a}}$ & $4^{a}$ & $5^{a}$ & $6^{\mathbf{a}}$ \\
\hline \multirow{6}{*}{ Pele } & Grau 0 & 11 & 4 & 1 & - & - & - \\
\hline & Grau 1 & 2 & 8 & 6 & 2 & 2 & 1 \\
\hline & Grau 2 & - & 1 & 5 & 6 & 7 & 9 \\
\hline & Grau 3 & - & - & 1 & 5 & 3 & 1 \\
\hline & Grau 4 & - & - & - & - & 1 & 1 \\
\hline & Total & 13 & 13 & 13 & 13 & 13 & 12 \\
\hline \multirow{6}{*}{ Mucosa } & Grau 0 & 11 & 2 & - & - & - & - \\
\hline & Grau 1 & 1 & 7 & 6 & 1 & 1 & 2 \\
\hline & Grau 2 & 1 & 4 & 3 & 7 & 6 & 4 \\
\hline & Grau 3 & - & - & 4 & 4 & 4 & 4 \\
\hline & Grau 4 & - & - & & 1 & 2 & 2 \\
\hline & Total & 13 & 13 & 13 & 13 & 13 & 12 \\
\hline \multirow{6}{*}{$\begin{array}{l}\text { Glândulas } \\
\text { Salivares }\end{array}$} & Grau 0 & 11 & 6 & 1 & - & - & - \\
\hline & Grau 1 & 2 & 6 & 8 & 4 & 2 & - \\
\hline & Grau 2 & - & 1 & 3 & 8 & 10 & 2 \\
\hline & Grau 3 & - & - & 1 & 1 & - & 9 \\
\hline & Grau 4 & - & - & - & - & 1 & 1 \\
\hline & Total & 13 & 13 & 13 & 13 & 13 & 12 \\
\hline \multirow{6}{*}{$\begin{array}{l}\text { Faringe/ } \\
\text { Esófago }\end{array}$} & Grau 0 & 9 & 6 & 3 & 1 & 1 & 1 \\
\hline & Grau 1 & 3 & 5 & 7 & 6 & 4 & 3 \\
\hline & Grau 2 & 1 & 1 & 2 & 4 & 5 & 4 \\
\hline & Grau 3 & - & - & - & 1 & 2 & 3 \\
\hline & Grau 4 & - & - & 1 & 1 & 1 & 1 \\
\hline & Total & 13 & 13 & 13 & 13 & 13 & 12 \\
\hline \multirow{6}{*}{ Laringe } & Grau 0 & 11 & 10 & 9 & 8 & 8 & 7 \\
\hline & Grau 1 & 2 & 3 & 3 & 3 & 1 & 1 \\
\hline & Grau 2 & - & - & 1 & 2 & 4 & 3 \\
\hline & Grau 3 & - & - & - & - & - & 1 \\
\hline & Grau 4 & - & - & - & - & - & - \\
\hline & Total & 13 & 13 & 13 & 13 & 13 & 12 \\
\hline
\end{tabular}

Ao longo do período de avaliação observou-se entre a $3^{\mathrm{a}}$ e a $4^{\mathrm{a}}$ semana, a suspensão do tratamento em um doente do Grupo 2 (RapidArc ${ }^{\mathrm{TM}}$ ), por motivos não relacionados com o tratamento. Entre a $5^{\mathrm{a}}$ e a $6^{\mathrm{a}}$ semana, terminaram o tratamento 1 doente do Grupo 1 e 14 doentes do Grupo 2, perfazendo um total de 30 doentes que continuaram em avaliação na $6^{\mathrm{a}}$ semana. As tabelas 2 e 3 representam a evolução dos doentes avaliados em termos dos efeitos agudos desenvolvidos, nas estruturas em avaliação, ao longo 5/6 semanas de avaliação, consoante a técnica a que foram submetidos.

Para facilitar a interpretação dos dados, foram construídos gráficos de barras a partir das tabelas referidas anteriormente (Figura 1). Todos os doentes do Grupo 
Table 3: Results observed in Group 2 (RapidArc ${ }^{\mathrm{TM}}$ ) in different structures in evaluation during the treatment weeks

\begin{tabular}{|c|c|c|c|c|c|c|c|}
\hline \multirow{2}{*}{$\begin{array}{l}\text { Structure in } \\
\text { Evaluation }\end{array}$} & \multirow{2}{*}{ Grade } & \multicolumn{6}{|c|}{ Weeks of treatment } \\
\hline & & $\mathbf{1}^{\mathrm{a}}$ & $2^{a}$ & $3^{\mathbf{a}}$ & $4^{\mathrm{a}}$ & $5^{a}$ & $6^{\mathrm{a}}$ \\
\hline \multirow{6}{*}{ Skin } & Grade 0 & 32 & 15 & 5 & 1 & - & - \\
\hline & Grade 1 & 1 & 16 & 23 & 17 & 10 & 4 \\
\hline & Grade 2 & - & 2 & 3 & 11 & 18 & 12 \\
\hline & Grade 3 & - & - & 2 & 3 & 3 & 2 \\
\hline & Grade 4 & - & - & - & - & 1 & - \\
\hline & Total & 33 & 33 & 33 & 32 & 32 & 18 \\
\hline \multirow{6}{*}{ Mucosa } & Grade 0 & 28 & 5 & - & - & - & - \\
\hline & Grade 1 & 3 & 17 & 14 & 7 & 5 & 7 \\
\hline & Grade 2 & 2 & 10 & 14 & 16 & 13 & 10 \\
\hline & Grade 3 & - & 1 & 5 & 9 & 14 & 1 \\
\hline & Grade 4 & - & - & - & - & - & - \\
\hline & Total & 33 & 33 & 33 & 32 & 32 & 18 \\
\hline \multirow{6}{*}{$\begin{array}{c}\text { Salivary } \\
\text { Glands }\end{array}$} & Grade 0 & 23 & 11 & 6 & 4 & 4 & 2 \\
\hline & Grade 1 & 9 & 16 & 14 & 8 & 4 & 5 \\
\hline & Grade 2 & 1 & 6 & 13 & 20 & 24 & 11 \\
\hline & Grade 3 & - & - & - & - & - & - \\
\hline & Grade 4 & - & - & - & - & - & - \\
\hline & Total & 33 & 33 & 33 & 32 & 32 & 18 \\
\hline \multirow{6}{*}{$\begin{array}{l}\text { Pharynx / } \\
\text { Esophagus }\end{array}$} & Grade 0 & 24 & 11 & 8 & 6 & 3 & 1 \\
\hline & Grade 1 & 7 & 15 & 10 & 9 & 11 & 6 \\
\hline & Grade 2 & 2 & 5 & 12 & 15 & 13 & 6 \\
\hline & Grade 3 & - & 2 & 3 & 2 & 5 & 5 \\
\hline & Grade 4 & - & - & - & - & - & - \\
\hline & Total & 33 & 33 & 33 & 32 & 32 & 18 \\
\hline \multirow{6}{*}{ Larynx } & Grade 0 & 32 & 28 & 22 & 20 & 19 & 10 \\
\hline & Grade 1 & 1 & 5 & 10 & 9 & 10 & 6 \\
\hline & Grade 2 & - & - & 1 & 3 & 3 & 2 \\
\hline & Grade 3 & - & - & - & - & - & - \\
\hline & Grade 4 & - & - & - & - & - & - \\
\hline & Total & 33 & 33 & 33 & 32 & 32 & 18 \\
\hline
\end{tabular}

were observed as a result of both techniques. Therefore, the skin was the only structure where IMRT had different impact compared to RapidArc ${ }^{\mathrm{TM}}$. This is because the acute effects appeared faster in Group 2 but were much more evident in Group 1. Data analysis also revealed that patients who developed more severe effects were undergoing both chemotherapy and radiotherapy. In the mucosa, effects from both treatment techniques were observed in all patients from the $3^{\text {rd }}$ week onwards. However, as a result of the IMRT technique, 5 patients exhibited Grade 3 effects and 1 patient Grade 4 effects in the $4^{\text {th }}$ week which corresponds to a significant increase in high-grade effects. Furthermore, it was found that 4 patients in Group 2 did not show any effects in the salivary glands during the 6 weeks of treatment. Moreover, higher-grade effects (Grade 3 and 4) were only observed in Group 1. In the
Tabela 3: Resultados observados no Grupo 2 (RapidArc ${ }^{\mathrm{TM}}$ ), nas diferentes estruturas em avaliação durante as semanas de tratamento

\begin{tabular}{|c|c|c|c|c|c|c|c|}
\hline \multirow{2}{*}{$\begin{array}{c}\text { Estrutura } \\
\text { em Avaliação }\end{array}$} & \multirow{2}{*}{ Graus } & \multicolumn{6}{|c|}{ Semanas de tratamento } \\
\hline & & $1^{\mathrm{a}}$ & $2^{\mathrm{a}}$ & $3^{\mathrm{a}}$ & $4^{a}$ & $5^{a}$ & $6^{a}$ \\
\hline \multirow{6}{*}{ Pele } & Grau 0 & 32 & 15 & 5 & 1 & - & - \\
\hline & Grau 1 & 1 & 16 & 23 & 17 & 10 & 4 \\
\hline & Grau 2 & - & 2 & 3 & 11 & 18 & 12 \\
\hline & Grau 3 & - & - & 2 & 3 & 3 & 2 \\
\hline & Grau 4 & - & - & - & - & 1 & - \\
\hline & Total & 33 & 33 & 33 & 32 & 32 & 18 \\
\hline \multirow{6}{*}{ Mucosa } & Grau 0 & 28 & 5 & - & - & - & - \\
\hline & Grau 1 & 3 & 17 & 14 & 7 & 5 & 7 \\
\hline & Grau 2 & 2 & 10 & 14 & 16 & 13 & 10 \\
\hline & Grau 3 & - & 1 & 5 & 9 & 14 & 1 \\
\hline & Grau 4 & - & - & - & - & - & - \\
\hline & Total & 33 & 33 & 33 & 32 & 32 & 18 \\
\hline \multirow{6}{*}{$\begin{array}{l}\text { Glândulas } \\
\text { Salivares }\end{array}$} & Grau 0 & 23 & 11 & 6 & 4 & 4 & 2 \\
\hline & Grau 1 & 9 & 16 & 14 & 8 & 4 & 5 \\
\hline & Grau 2 & 1 & 6 & 13 & 20 & 24 & 11 \\
\hline & Grau 3 & - & - & - & - & - & - \\
\hline & Grau 4 & - & - & - & - & - & - \\
\hline & Total & 33 & 33 & 33 & 32 & 32 & 18 \\
\hline \multirow{6}{*}{$\begin{array}{l}\text { Faringe/ } \\
\text { Esófago }\end{array}$} & Grau 0 & 24 & 11 & 8 & 6 & 3 & 1 \\
\hline & Grau 1 & 7 & 15 & 10 & 9 & 11 & 6 \\
\hline & Grau 2 & 2 & 5 & 12 & 15 & 13 & 6 \\
\hline & Grau 3 & - & 2 & 3 & 2 & 5 & 5 \\
\hline & Grau 4 & - & - & - & - & - & - \\
\hline & Total & 33 & 33 & 33 & 32 & 32 & 18 \\
\hline \multirow{6}{*}{ Laringe } & Grau 0 & 32 & 28 & 22 & 20 & 19 & 10 \\
\hline & Grau 1 & 1 & 5 & 10 & 9 & 10 & 6 \\
\hline & Grau 2 & - & - & 1 & 3 & 3 & 2 \\
\hline & Grau 3 & - & - & - & - & - & - \\
\hline & Grau 4 & - & - & - & - & - & - \\
\hline & Total & 33 & 33 & 33 & 32 & 32 & 18 \\
\hline
\end{tabular}

1 desenvolveram efeitos na Pele a partir da $4^{\mathrm{a}}$ semana, enquanto que no Grupo 2, esta situação só se verificou a partir da $5^{\text {a }}$ semana. Em ambas as técnicas surgiram efeitos de Grau 4 na $5^{\text {a }}$ semana. Portanto, a pele foi a única estrutura em que a IMRT se diferenciou relativamente ao RapidArc ${ }^{\mathrm{TM}}$, no que diz respeito ao aparecimento dos efeitos, já que estes surgiram mais rapidamente no Grupo 2, mas com maior severidade no Grupo 1. Pela análise dos dados, constatou-se, também, que os doentes que desenvolveram efeitos de maior severidade encontravam-se em esquemas de Radioterapia e Quimioterapia concomitante.

Relativamente aos efeitos observados na Mucosa, em ambas as técnicas verificaram-se efeitos em todos os doentes a partir da $3^{\mathrm{a}}$ semana. No entanto, na técnica de IMRT, a partir da $4^{\mathrm{a}}$ semana observou-se 5 doentes com Grau 3 e 1 doente com Grau 4, o que corresponde 
pharynx / esophagus, Grade 4 effects were registered from the $3^{\text {rd }}$ week in a patient in Group 1 who was also undergoing CT. Regarding the RapidArc ${ }^{\mathrm{TM}}$ technique, Grade 3 effects were verified from week 2 and developed progressively until the end of treatment.

In the larynx, Grade 2 effects resulting from both techniques were identified from week 3 . Grade 3 effects were exclusive to the IMRT technique and were observed in week 6 .
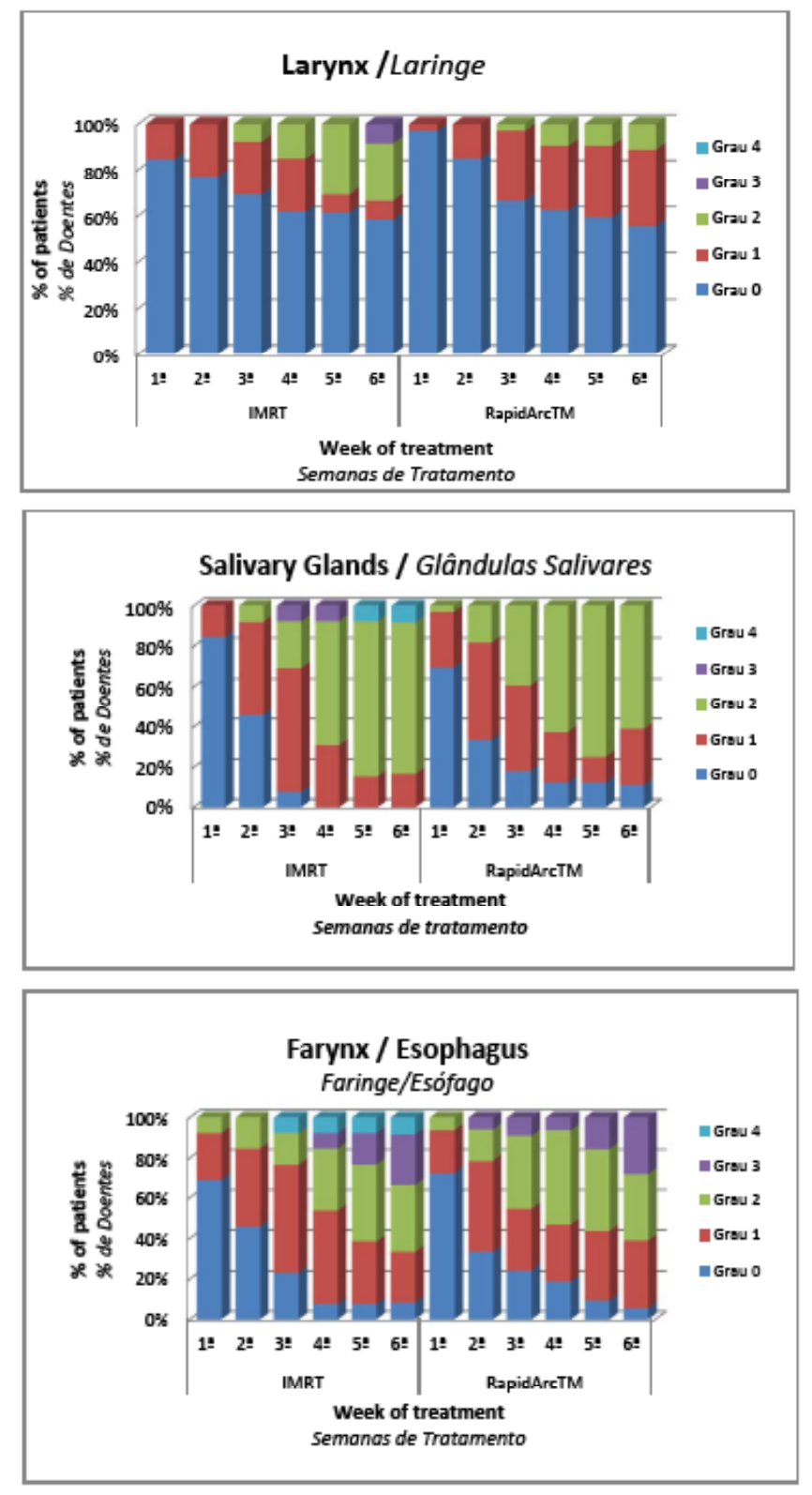

a um aumento significativo dos efeitos de alto Grau. Por sua vez, verificou-se no Grupo 2 que 4 indivíduos da amostra não manifestaram qualquer tipo de efeito ao longo das 6 semanas de tratamento, ao nível das Glândulas Salivares. Além disso, os efeitos de maior Grau (Grau 3 e 4) apenas se observaram no Grupo 1.

$\mathrm{Na}$ Faringe/Esófago existe um registo de efeitos de Grau 4 a partir da $3^{\text {a }}$ semana, num doente do Grupo 1 que estava a realizar QT concomitante. Na técnica de RapidArc $^{\mathrm{TM}}$ verificaram-se efeitos de Grau 3 (a partir da $2^{\mathrm{a}}$ semana), evoluindo de uma forma progressiva até ao final do tratamento.

Ao nível da Laringe, em ambas as técnicas identificaram-se efeitos de Grau 2 a partir da $3^{\mathrm{a}}$ semana e efeitos de Grau 3, unicamente, na técnica de IMRT, com registos na $6^{\mathrm{a}}$ semana.
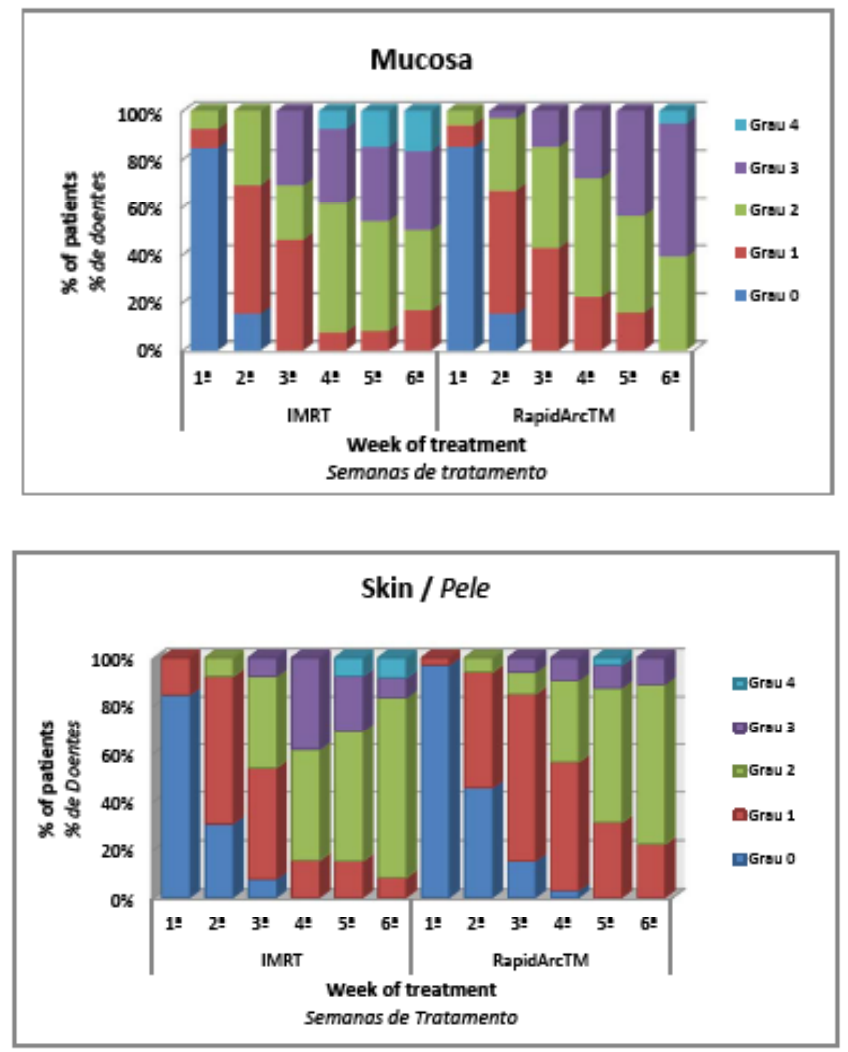

Figure 1 - Representation of the results obtained in the evaluation of effects on Skin, Mucosa, Salivary Glands, Pharynx / Esophagus and Larynx, normalized to the total number of patients by technique.

Figura 1 - Representação dos resultados obtidos na avaliação dos efeitos na Pele, Mucosa, Glândulas Salivares, Faringe/ Esófago e Laringe, normalizados ao número total de doentes por técnica. 


\section{Discussion}

The manifestation of acute effects may be influenced by several factors, including factors inherent to the patient (such as their intrinsic radiosensibility) or external factors to the patient (such as the technique, fractionation, dose and associated therapies). By reviewing the skin results, some patients studied developed effects in the $1^{\text {st }}$ week of evaluation. These observations are supported by the study of Wood et al. in 2006, an investigation that also found similar results. The Grade 3 and Grade 4 effects observed were verified in patients undergoing concomitant $\mathrm{CT}$. This supports the idea that chemotherapy influences the tolerance and individual radiosensitivity of each patient's tissues.

In the evaluation of the mucosa, it was observed that some patients revealed Grade 2 and Grade 1 effects in the $1^{\text {st }}$ week of treatment. This result was also observed by Vissink and his group in 2003, whose investigation used the IMRT technique. Towards the $3^{\text {rd }}$ week of treatment, symptoms intensified and patients presented Grade 3 effects where pseudomembranes and tissue ulceration were observed in both techniques.

Scorsetti and his group in 2010 initiated a clinical experiment with the RapidArc ${ }^{\mathrm{TM}}$ technique involving 45 patients with HNC. They also described Grade 3 effects in the mucosa, but only in the $5^{\text {th }}$ week of treatment. Regarding the IMRT technique, Grade 4 effects were observed from the $4^{\text {th }}$ week. In comparison, the Rapi$\mathrm{dArc}^{\mathrm{TM}}$ technique provoked effects in mucosa only in week 6. Patients from both treatment techniques were also undergoing combined treatment with chemotherapy, which could have influenced the toxicity increase in the normal tissues ${ }^{10,18,5,6}$.

With regards to the salivary glands results, we have observed that all IMRT patients demonstrated effects in the $4^{\text {th }}$ week of treatment, with some patients experiencing high-degree effects. Not all patients undergoing treatment with the RapidArc ${ }^{\mathrm{TM}}$ technique developed effects and the highest severity effect registered in these patients was Grade 2. Doornaert and his group in 2011 also observed Grade 2 effects, as the most severe effects in salivary glands in a study of 31 patients with HNC.

In realtion to the pharynx/ esophagus, Grade 4 effects were observed in 1 patient in the $3^{\text {rd }}$ week exclusively with IMRT. This patient was also undergoing CT. Grade 3 was the highest degree effect observed in the Rapi$\mathrm{dArc}{ }^{\mathrm{TM}}$ and was registered in the $2^{\text {nd }}$ week in 5 patients of a total of 32 in 5 weeks. In general, in radiotherapy patients with $\mathrm{HNC}$, usually between $20 \%$ and $30 \%$ (mean) of them use artificial feeding due to RT effects such as dysphagia and odynophagia ${ }^{13}$. In this study, we

\section{Discussão}

A manifestação dos efeitos agudos pode ser influenciada por diversos fatores, nomeadamente, fatores inerentes ao próprio paciente (como é o caso da radiossensibilidade intrínseca) ou fatores externos ao doente (como a técnica utilizada, fracionamento, dose e terapias associadas). $\mathrm{Na}$ análise dos resultados da Pele, logo na $1^{\mathrm{a}}$ semana de avaliação, alguns dos doentes, em estudo, desenvolveram efeitos ao nível desta estrutura, o que vai de encontro ao estudo de Wood et al., em 2006, numa investigação em que também observaram resultados semelhantes.

Os efeitos de Grau 3 e 4 observados foram verificados em doentes que se encontravam a realizar QT concomitante, o que vem de certo modo apoiar a influência dos quimioterápicos na tolerância e a radiossensibilidade individual dos tecidos de cada doente.

$\mathrm{Na}$ avaliação da Mucosa, observaram-se alguns doentes com efeitos de Grau 1 e Grau 2, logo $1^{a}$ semana de tratamento, situação também observada por Vissink e o seu grupo em 2003, numa investigação com a técnica de IMRT. Perto da $3^{\text {a }}$ semana de tratamento, os sintomas observados intensificaram-se, com a formação de pseudomembranas e ulceração dos tecidos, em ambas as técnicas, verificaram-se doentes com efeitos de Grau 3 logo na $3^{\text {a }}$ semana. Scorsetti e o seu grupo, em 2010, numa experiencia clínica de iniciação com a técnica de RapidArc ${ }^{\mathrm{TM}}$, em 45 doentes com CCP, descreveram também efeitos de Grau 3 na Mucosa, mas somente na $5^{\text {a }}$ semana de tratamento. Na técnica de IMRT, a partir da $4^{\mathrm{a}}$ semana, observaram-se efeitos de Grau 4, enquanto na técnica de RapidArc ${ }^{\mathrm{TM}}$ só foram encontrados efeitos a este nível na $6^{\mathrm{a}}$ semana. Em ambas as técnicas, estes doentes encontravam-se, também, a realizar esquemas de QT concomitante, facto que poderá ter influenciado no aumento da toxicidade dos tecidos normais ${ }^{10,18,5,6}$.

Quanto à avaliação dos resultados ao nível das Glândulas Salivares, todos os doentes de IMRT manifestaram efeitos a partir da $4^{\mathrm{a}}$ semana, observando-se efeitos de alto Grau em alguns doentes. Na técnica de RapidArc ${ }^{\mathrm{TM}}$ nem todos os doentes desenvolveram efeitos e verificou-se que o efeito de maior Grau de severidade foi o Grau 2. Doornaert e o seu grupo, em 2011, também verificaram efeitos de Grau 2, como efeitos de maior severidade, nas Glândulas Salivares num estudo realizado em 31 doentes com CCP, submetidos a RapidArc ${ }^{\mathrm{TM}}$, observando-se uma similaridade de resultados com os apresentados ao longo deste estudo.

Nos efeitos ao nível da Faringe/Esófago, verificaram-se, na $3^{\text {a }}$ semana, efeitos de Grau 4, exclusivamente, com a IMRT, num doente e que se encontravam também a realizar QT concomitante. Com a técnica de RapidArc ${ }^{\mathrm{TM}}$, o efeito mais exacerbado foi o Grau 3, observado a partir da $2^{\text {a }}$ semana, num total de 5 doentes em 32 na $5^{\text {a }}$ semana. 
confirmed that $35 \%$ of our observed patients used artificial feeding, a slightly higher percentage compared to the mean.

Regarding the larynx, Group 1 patients showed cases of the most severe effects, which leads to the attributing of a higher co-morbidity for these patients.

On the other hand, we observed a regression of the effects in some of patients studied, in certain areas under evaluation. In particular in the skin, mucosa, pharynx / esophagus and larynx, mostly from the $5^{\text {th }}$ week of evaluation onwards. This is related to the installation of medical and nursing care and monitoring provided for the patient. Regarding the comparison of acute effects in both techniques, those effects produced by the RapidArc ${ }^{\mathrm{TM}}$ technique emerged more rapidly than those produced by the IMRT technique; the effects were already registed in weeks 1-2 of treatment. On the other hand, effects caused by the RapidArc ${ }^{\mathrm{TM}}$ technique were less severe when compared with IMRT.

\section{Conclusion}

The acute effects of RT in patients with HNC have a complex multifactorial manifestation. Regarless of whether the technique used was IMRT or the Rapi$\mathrm{dArc}{ }^{\mathrm{TM}}$, in this study a general progression of effects was detected over the weeks of treatment. Evaluation of the effects and data analysis allowed us to verify that the acute effects obtained with the RapidArc ${ }^{\mathrm{TM}}$ technique appear earlier than with the IMRT technique. More pronounced high-degree effects were registered with the IMRT technique. The evaluation of acute effects in patients with HNC plays an important role during the whole radiotherapy treatment, since the degree of severity of these effects may justify interruption/ suspension of the treatment itself. In this context, and based on data collected and analyzed, future studies need to be developed with a larger sample and introducing new variables. The aim should be to consolidate and validate the results obtained here in addition to creating new clinical interventions and personalized integrated care strategies to contribute to improving the radiotherapy oncologic patients' quality of life.
A utilização de suportes de alimentação artificial pode ser requerida por doentes, o que normalmente acontece entre $20 \%$ e $30 \%$ dos doentes em tratamento de Radioterapia ${ }^{13}$. No presente estudo, $35 \%$ do total de doentes, 16 doentes, utilizaram suportes artificiais de alimentação, por dificuldades de ingestão e prevenção de quadros de malnutrição, despoletados pelo tratamento.

Nos resultados obtidos ao nível da Laringe, observou-se que foi no Grupo 1 que se verificaram os efeitos de maior severidade, pelo que se atribui uma maior co-morbilidade aos doentes no tratamento.

Em alguns doentes em estudo, por sua vez, observou-se uma regressão dos efeitos ao tratamento em determinadas estruturas em avaliação, nomeadamente na Pele, Mucosa, Faringe/Esófago e Laringe, principalmente a partir da $5^{\mathrm{a}}$ semana de avaliação. Este último facto encontra-se relacionado com o acompanhamento e início dos cuidados médicos e de enfermagem prestados ao doente. Já na comparação da manifestação dos efeitos agudos com as duas técnicas, verificou-se que os efeitos com a técnica de RapidArc ${ }^{\mathrm{TM}}$ surgiram mais rapidamente, do que com a técnica de IMRT, com um registo de efeitos logo na $1^{\mathrm{a}}$ ou $2^{\mathrm{a}}$ semana de tratamento. Mas, em contrapartida, os efeitos com a técnica de RapidArc ${ }^{\mathrm{TM}}$, manifestaram-se em geral com um grau de severidade inferior, quando comparados com a IMRT.

\section{Conclusão}

Os efeitos agudos da RT em doentes com CCP manifestam-se de forma complexa e multifatorial. Independentemente da técnica utilizada, IMRT ou RapidArc ${ }^{\mathrm{TM}}$, observou-se uma progressão, em geral, dos efeitos ao longo das semanas de tratamento. Após a avaliação e análise dos dados, verificou-se que os efeitos agudos obtidos com a técnica de RapidArcTM surgiram mais precocemente, quando comparados com técnica de IMRT. Por outro lado, com a técnica de IMRT registaram-se efeitos mais exacerbados, de alto Grau. A avaliação dos efeitos agudos nos doentes com CCP assume um papel importante durante todo o tratamento com RT, uma vez que o grau de severidade destes efeitos pode justificar uma interrupção/suspensão do próprio tratamento. Neste contexto e com base nos dados recolhidos e analisados, há a necessidade de desenvolver trabalhos futuros, com uma amostragem maior e introdução de novas variáveis, não só para consolidar e validar os resultados obtidos nesta investigação, como também permitir a criação de novas estratégias de intervenção clinica e de cuidados personalizados e integrados, que contribuam para melhoria da qualidade de vida do doente submetido a RT e do doente Oncológico. 


\section{Acknowledgments}

To Chief-nurse Amélia Osório of the Nursing Service of Radiotherapy of IPO-Porto, for all the support provided and the willingness and spirit of helpfulness that she always demonstrated.

To the Radiotherapy Department of IPO-Porto Director, Dr. Helena Pereira, for her cooperation and support.

To Dr. Marisa Lobão, for the amazing cooperation in the data acquisition, the willingness and interest that she has always shown.

To Dr. Vera Castro, for her distinguished cooperation in data acquisition, her interest shown throughout the development of the study, and her precious opinions and knowledge shared along the whole process. To Dr. Maria José Bento, for all the support provided in data processing.

To Therapists Fatima Borges and Luciano Gomes, for their receptiveness to the various questions that have emerged throughout this study.

\section{Conflict of interests}

The authors declare that there are no financial and personal relationships that could be viewed as presenting a potential conflict of interests.

\section{Agradecimentos}

À Enfermeira Chefe do Serviço de Radioterapia, Enfermeira Amélia Osório, por todo o apoio prestado na concretização deste estudo, pela disponibilidade e espírito de ajuda que sempre revelou.

À Diretora do Serviço de Radioterapia, a Dr ${ }^{\mathrm{a}}$. Helena Pereira, pela cooperação e apoio na concretização deste estudo.

À Dra . Marisa Lobão, pela exímia cooperação na aquisição dos dados, pela disponibilidade e interesse que sempre demonstrou.

À Dra ${ }^{\mathrm{a}}$. Vera Castro, pela distinta cooperação na aquisição de dados, interesse demonstrado ao longo de todo o desenvolvimento do estudo, preciosas opiniões e conhecimentos que foi partilhando ao longo de todo este processo.

À Dra ${ }^{\mathrm{a}}$. Maria José Bento, por todo o apoio prestado no tratamento dos dados.

À Terapeuta Fátima Borges e ao Terapeuta Luciano Gomes, por toda a disponibilidade que sempre revelaram perante as mais variadas dúvidas que foram surgindo ao longo deste estudo.

\section{Conflito de interesses}

Os autores declaram que não existem quaisquer relações pessoais ou financeiras que possam ser consideradas como um potencial conflito de interesses. 


\section{References / Referências}

(1). Argiris A., Karamouzis M., Raben D., Ferris R. Head and neck cancer. Lancet. 2008; 371 : p.1695-709.

(2). Barbosa B. Implicações Radiobiológicas da IMRT em Carcinomas da Próstata. Porto. Dezembro de 2010, p. 3-61.

(3). Bhide S.A., Nutting C.M. Advances in radiotherapy for head and neck cancer. Elsiver: Oral Oncology 46. 2010; p. 439-441.

(4). Chen A. M., Li B., Farwell D. G., Marsano J., Vijayakumar S., PurdymJ. Improved Dosimetric and Clinical outcomes with intensitymodulated Radiotherapy for Head-and-Neck Cancer of unknown primary origin. Elsevier : Int. J. Radiation Oncology Biol. Phys., Vol. $79, \mathrm{n}^{\circ} 3.2011$; p. $756-762$

(5). Doornaert P., Verbakel W. F. A. R., Bieker M., Slotman B. J., Senan S. RapidArc Planning and Delivery in patients with Locally Advanced Head-and-Neck Cancer undergoing Chemoradiotherapy. I. J. Radiation Oncology and Biology and Physics Volume 79, Number 2. 2011; p. 429-435.

(6). Doornaert P., Verbakel W., Rietveld D., Slotman B. J., Senan S. Sparing the contralateral submandibular gland without compromising PTV coverage by using Volumetric Modulated Arc Therapy”. Radiation Oncology. 2011; p. 6-74.
(7). Hussain Q. M., Ul-Alam M. Head and Neck Cancer- An Overview. The Journal of Teachers Association RMC, Rajshahi, TAJ June 2007; Volume 20 Number1.

(8). Kisling K. Volumetric Modulated Arc Therapy Evaluation with the Radiobiological Physics Center Head and Neck Phantom. UT GSBS Dissertations and Theses. Paper 70; 2010.

(9). Movsas B., Vikram B., Hauer-Jensen M., Moulden J., Baschs E., Brown S., Kachnics L. A., Dicker A. P., Coleman C. N., Okunieffs P., Decreasing the Adverse Effects of Cancer Therapy: National Cancer Intitute Guidance of Clinical Develepment of Radiotion Injury Mitigotors. AACR. 2011 [cited 1 February 2012]. Available from: clincancerres.aacrjournals.org

(10).Murphy B. A., Gilbert J. Cancer Patients Treated with Radiation: Assessment, Sequelae, and Rehabilitation. Seminars in Radiation Oncology, ELSEVIER. 2009.

(11).Perez C. A, Brady L. W. Perez and Brady's Principles and Practice of Radiation Oncology. 5th edition. s.1. : Lippincott Williams \& Wilkins; 2008

(12).Suntharalingan N., Podgorsak E. B., Hendry J.H. Basic Radiobiology; 2010, p. 483-503.
(13).Vissink A., Jansma J., Spijkervet F.K.L., Burlagez F.R., Coppes R.P. Oral Sequelae of Head na Neck Radiotherapy [Internet]. SAGE. 2003[cited 24 January 2012].

(14).Trotti A., Colevas D., Setser A., Rusch V., Jaques D., Budach V., Langer C., Murphy B., Cumberlin R., Coleman, C. N., Rubin P. CTCAE v3.0: Development of a Comprehensive Grading System for the Adverse Effects of Cancer Treatment. Seminars in Radiation Oncology, Vol 13, nº 3. 2003; p. 176-181

(15).Wood S. Jr, Woloschak G.E. Radiation Toxicity: A Pratical Guide. Springer, Library of Congress; 2006.

(16).Radiation Therapy Oncology Group (RTOG). Acute Radiation Morbidity Scoring; Criteria [Online]:http://www.rtog.org/ResearchAssociates/AdverseEventReporting/AcuteRadiationMorbidityScoringCriteria.aspx.

(17).Trotti A. Toxicity in Head and Neck Cancer: A review of trends and issues.Elsevier Science Inc: Int. J. Radiation Oncology Biol. Phys., Vol. 47, $n^{\circ}$ 1. 2000; p. 1-12.

(18).Scorsetti M., Fogliata A., Castiglioni S. Bressi C., Bignardi M., Navarria P., Mancosu P., Cozzi L., Pentimalli S., Alongi F., Santoro A. Early clinical experience with Volumetric Modulated Arc Therapy in Head and Neck Cancer patients. Radiation Oncology. 2010; p. 5-93. 